\title{
Differential physiological, transcriptomic and metabolomic responses of Arabidopsis leaves under prolonged warming and heat shock
}

Li Wang ${ }^{1,2+}$, Kai-Biao Ma ${ }^{1 \dagger}$, Zhao-Geng Lu', Shi-Xiong Ren ${ }^{1}$, Hui-Ru Jiang ${ }^{1}$, Jia-Wen Cui ${ }^{1}$, Gang Chen ${ }^{3}$, Nian-Jun Teng ${ }^{4}$, Hon-Ming Lam² ${ }^{2^{*}}$ and Biao Jin ${ }^{1 *}$ (D)

\begin{abstract}
Background: Elevated temperature as a result of global climate warming, either in form of sudden heatwave (heat shock) or prolonged warming, has profound effects on the growth and development of plants. However, how plants differentially respond to these two forms of elevated temperatures is largely unknown. Here we have therefore performed a comprehensive comparison of multi-level responses of Arabidopsis leaves to heat shock and prolonged warming.

Results: The plant responded to prolonged warming through decreased stomatal conductance, and to heat shock by increased transpiration. In carbon metabolism, the glycolysis pathway was enhanced while the tricarboxylic acid (TCA) cycle was inhibited under prolonged warming, and heat shock significantly limited the conversion of pyruvate into acetyl coenzyme A. The cellular concentration of hydrogen peroxide $\left(\mathrm{H}_{2} \mathrm{O}_{2}\right)$ and the activities of antioxidant enzymes were increased under both conditions but exhibited a higher induction under heat shock. Interestingly, the transcription factors, class A1 heat shock factors (HSFA1s) and dehydration responsive elementbinding proteins (DREBs), were up-regulated under heat shock, whereas with prolonged warming, other abiotic stress response pathways, especially basic leucine zipper factors (bZIPs) were up-regulated instead.
\end{abstract}

Conclusions: Our findings reveal that Arabidopsis exhibits different response patterns under heat shock versus prolonged warming, and plants employ distinctly different response strategies to combat these two types of thermal stress.

Keywords: Arabidopsis thaliana, Climate warming, Heat stress, Omics, Photosynthesis, Respiration, Transcription factors

\section{Background}

As a result of climate warming, plants, due to their sessile lifestyle, need to develop a set of responses to adapt to the increasing temperature. Past studies on elevatedtemperature treatments could be generalized into two

\footnotetext{
* Correspondence: bjin@yzu.edu.cn; honming@cuhk.edu.hk

${ }^{\dagger}$ Li Wang and Kai-Biao Ma contributed equally to this work.

${ }^{2}$ Center for Soybean Research of the State Key Laboratory of

Agrobiotechnology and School of Life Sciences, The Chinese University of Hong Kong, Hong Kong, SAR, China

${ }^{1}$ College of Horticulture and Plant Protection, Yangzhou University, Yangzhou 225009, China

Full list of author information is available at the end of the article
}

categories: short-term intensive heat (also known as heat shock) and prolonged warming. Traditionally, for heat shock treatment, plants are subjected to a temperature that is much higher (such as $10-15^{\circ} \mathrm{C}$ above ambient) than their optimal threshold within a very short time (from several minutes to a few hours) [1]. On the other hand, prolonged warming is normally simulated by exposing plants to a moderately elevated temperature (such as $2-5^{\circ} \mathrm{C}$ above their optimal temperature range) for several days, weeks, or even the whole growing season $[2,3]$.

Previous studies on prolonged warming mainly investigated the phenology, reproduction and productivity,

(c) The Author(s). 2020 Open Access This article is distributed under the terms of the Creative Commons Attribution 4.0 International License (http://creativecommons.org/licenses/by/4.0/), which permits unrestricted use, distribution, and 
growth and development, and biomass accumulation [36], at the community, population and species levels, on species varying from grasses to trees $[2,7]$, while a few others examined the cellular, physiological and metabolomic responses [8-10]. However, the comprehensive analyses of responses to prolonged warming are rare.

In contrast, the physiological and molecular mechanisms involved in the responses to heat shock have been extensively studied in plants. Generally, heat shock reduces the photosynthetic and respiratory activities and diminishes productivity [9]. Intense heat induces structural and functional changes in the thylakoid membranes in photosynthetic apparatus, as a result of the production of reactive oxygen species (ROS) causing damage to a wide range of cellular components [11]. In response to heat shock, plants accelerate the production of heat shock proteins (HSPs) and accumulate responsive metabolites [12]. The roles of master transcriptional regulator HSFA1s and several other transcription factors, have been uncovered in the heat shock-related signaling pathways [13, 14]. However, very little is known about such regulatory mechanisms in prolonged warming responses. In particular, no study has focused on a systematic comparison of plant responses between prolonged warming and heat shock, despite the frequent occurrence of both in nature.

Leaves are the main vegetative organs that directly sense the changes in ambient temperature, and they can express phenotypically plastic responses to environmental temperature changes [15]. Moreover, leaf photosynthesis and transpiration, as the basis of plant growth and development, are susceptible to temperature changes [9]. Consequently, experiments on the effects of elevated temperatures on leaves will provide a better understanding of plant responses to heat stress. Therefore, we compared the physiological, transcriptomic and metabolomic responses of Arabidopsis leaves between prolonged warming and heat shock, and interpret these results in the contexts of their effects on photosynthesis and respiration, as well as the underlying transcriptional regulation.

\section{Results}

\section{Physiological and biochemical changes}

Arabidopsis thaliana plants were grown under control (CK), prolonged warming (PW) and heat shock (HS) treatments, and the leaves at the rosette growth stage were sampled (Fig. 1a, b). The stomatal conductance decreased under prolonged warming (Fig. 1c). However, the transpiration rate increased significantly under heat shock (Fig. 1d). Compared to control and heat shock, photosynthetic rate was decreased by prolonged warming (Fig. 1e). Similarly, prolonged warming had more pronounced effects on both $\mathrm{qP}$ and $\mathrm{qN}$, by decreasing $\mathrm{qP}$ and raising $\mathrm{qN}$ (Fig. 1f, g). However, there is no significant difference in $\mathrm{qP}$ and $\mathrm{qN}$ between control and heat shock (Fig. 1f, g).

Compared to control and prolonged warming, the level of soluble proteins was significantly lowered in heat shock (Fig. 1h). On the other hand, heat shock produced a significantly larger increase in both soluble sugars and hydrogen peroxide than prolonged warming (Fig. 1i, j). The activities of superoxide dismutase (SOD), catalase (CAT) and peroxidase (POD) in heat shock were significantly higher than those in prolonged warming (Fig. 1k, l, m).

\section{Metabolite changes}

Using GC-MS, we identified 181 metabolites that were significantly affected under prolonged warming and heat shock. Using principal component analysis (PCA) and orthogonal projection to latent structure with discriminant analysis (OPLS-DA), we separated these metabolites between experimental groups (Additional files 1: Figure S1a-d), and narrowed them down to 34 different metabolites (VIP $>1$ and $p<0.05$ ). The metabolome view map revealed that the enriched pathways $(p<0.05)$ between prolonged warming and heat shock were those involved in the citrate cycle, and glyoxylate and dicarboxylate metabolism (Fig. 2a).

The major metabolites differently accumulated among three treatments were listed in Fig. 2b. From the overall metabolite scenario, there was no common metabolites between heat shock vs $\mathrm{CK}$ and prolonged warming vs $\mathrm{CK}$, indicating that these two types of heat stresses led to completely differential trends of metabolite changes. For example, sorbitol was significantly increased in prolonged warming, while it was not found in heat shock. Some carbohydrate conjugates (such as DL-dihydrosphingosine, mannose, methyl-beta-D-galactopyranosic, and phenyl-beta-Dglucopyranoside) were decreased in heat shock (compared to CK), while they showed no changes in prolonged warming (compared to CK). Furthermore, many metabolites involved in intermediates of the TCA cycle such as fumaric acid, L-malic acid and citric acid were significantly decreased in prolonged warming (PW vs HS) (Fig. 2b).

\section{Differentially expressed genes (DEGs) associated with photosynthesis}

We generated RNA-Seq data from the leaves and obtained clean reads from three biological replicates each of CK $(102,596,706)$, prolonged warming $(77,761,052)$, and heat shock $(80,456,340)$ treatment, respectively (Additional files 4: Table S1). We further carried out differential expression analysis (Additional files 2: Figure S2a). Based on KEGG (Kyoto Encyclopedia of Genes and Genomes) enrichment analysis, genes involved in ribosome, photosynthesis, antenna proteins, and citrate cycle were enriched in both prolonged warming and heat shock (Additional files 2: Figure S2b, c, red arrows). On 
(a)

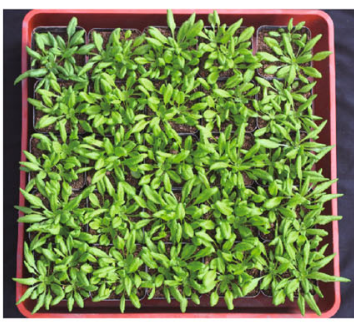

(c)

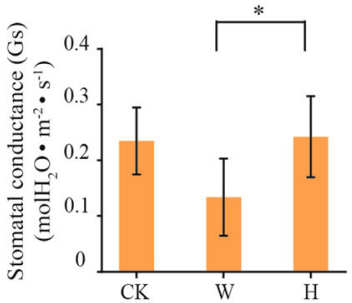

(f)

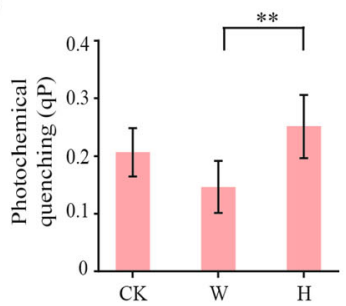

(i)

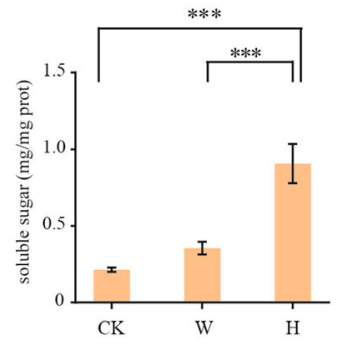

(I)

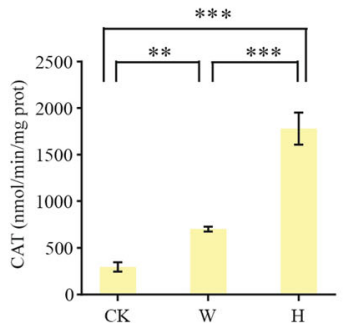

(b)

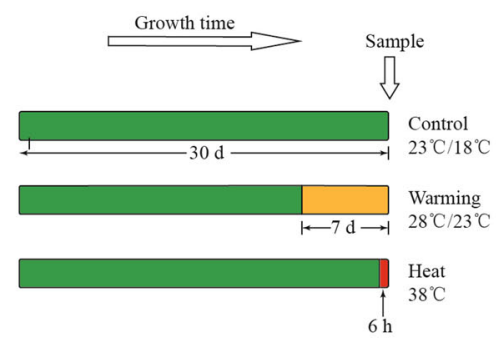

(e)

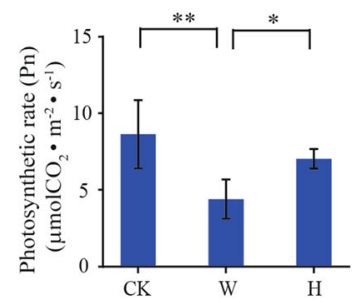

(h)

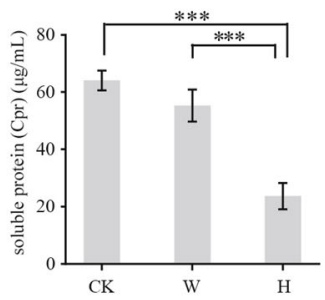

(j)

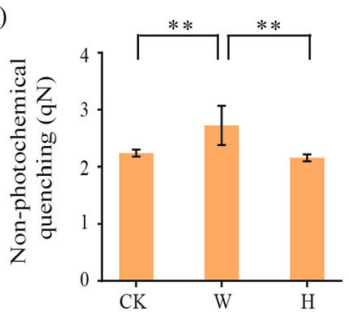

(k)
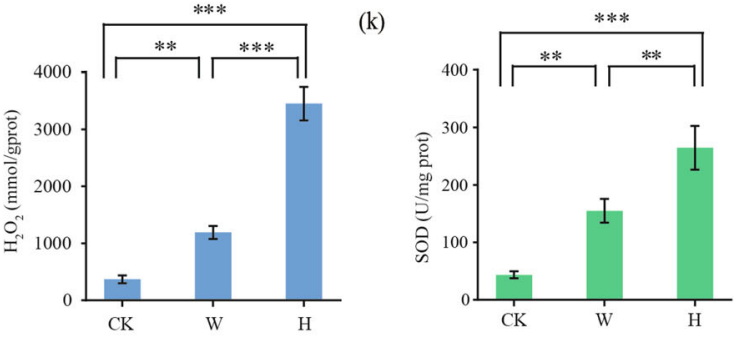

(m)

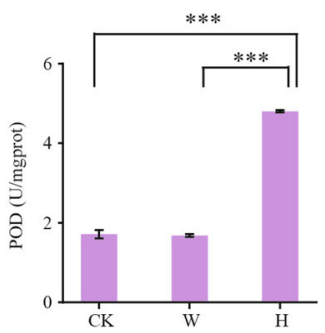

Fig. 1 Physiological characteristics of $A$. thaliana under different elevated temperature treatments. a Typical A. thaliana plants at the rosette growth stage 30 days after sowing. b The timeline for control (CK), prolonged warming (PW) and heat shock (HS) treatments showing a relatively prolonged period of warming (orange) for 7 days and a short-term heat shock (red) for 6 h. c Stomatal conductance, (d) transpiration rate, (e) photosynthetic rate, (f) photochemical quenching (qP), and (g) non-photochemical quenching (qN) were measured with a LI-6400XT Portable Photosynthesis System. $\mathbf{h}$ Soluble proteins, (i) soluble sugars, (j) hydrogen peroxide, the activities of (k) catalase (CAT), (I) superoxide dismutase $(\mathrm{SOD})$ and $(\mathbf{m})$ peroxidase (POD) in the leaves were determined at the end of the elevated temperature treatments. CK: control; PW: prolonged warming; HS: heat shock. Error bars represent mean \pm S.D. (c)-(g), $n=8,(\mathbf{h}-\mathbf{m}), n=3, *^{* * * * * *}: p<0.05 / 0.01 / 0.001$, respectively

the other hand, the pathways of porphyrin and chlorophyll metabolism and the biosynthesis of unsaturated fatty acids were significantly enriched in prolonged warming compared to heat shock (Additional files 2:
Figure S2d, red arrows). Based on GO (gene ontology) enrichments analysis, heat shock resulted in the enrichment of genes associated response to stimulus, response to stress, cellular component and membrane 


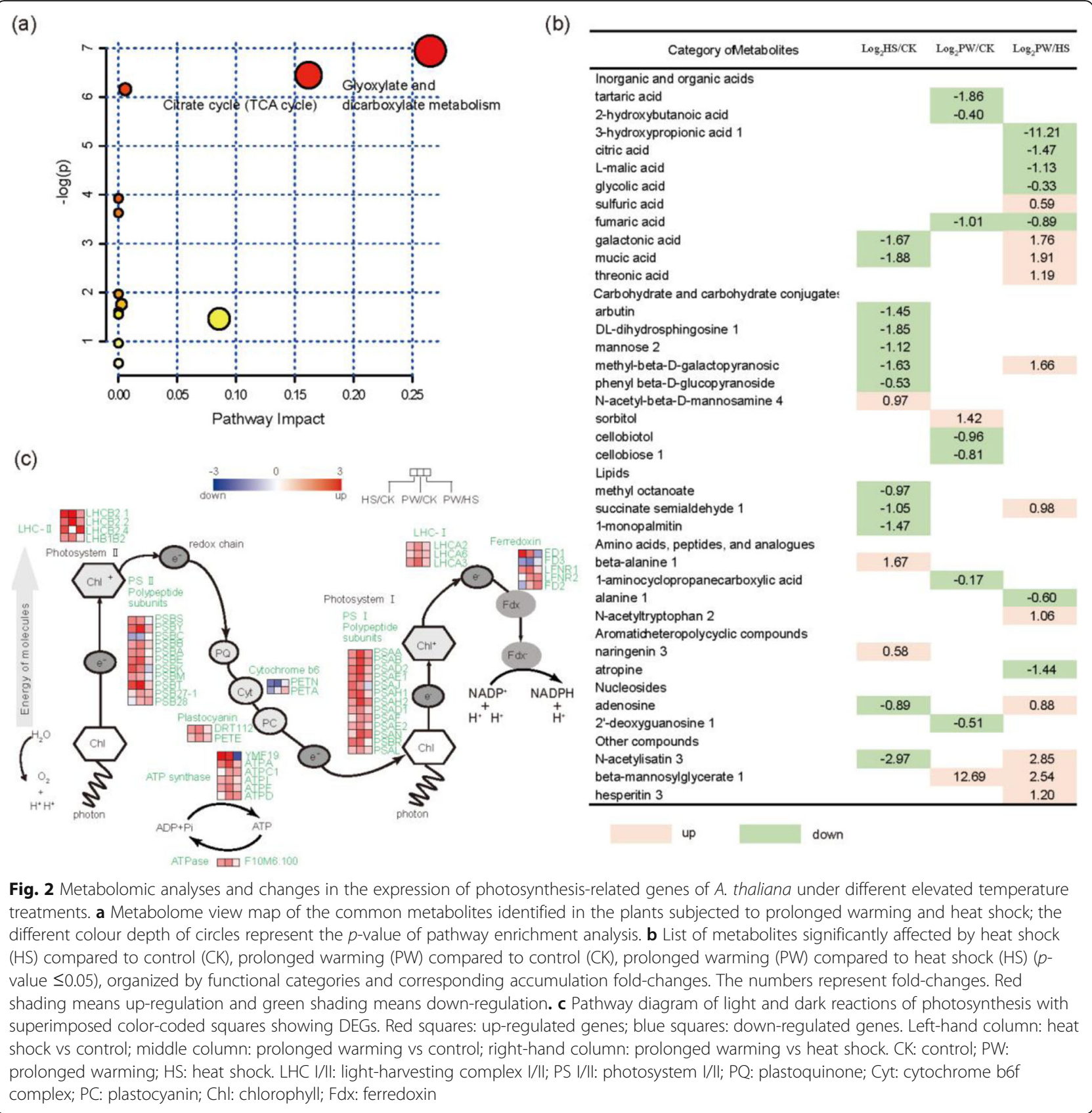

(Additional files 3: Figure S3a, arrows), while heat shock resulted in the enrichment of genes associated with response to abiotic stimulus, chloroplast, plastid, cytoplasm, intracellular part, photosynthesis and light reaction (Additional files 3: Figure S3b, arrows). Between the two elevated temperature treatments (prolonged warming vs heat shock), genes associated with response to stimulus, response to stress, chloroplast, plastid, cytoplasm and cytoplasmic part were enriched (Additional files 3: Figure S3c, arrows).

Informed by our KEGG and GO enrichment results, we further investigated DEGs associated with the photosynthetic electron transport system. We identified four DEGs involved in light harvesting complex II (LHC II), three DEGs encoding light harvesting complex I (LHC I), 11 DEGs related to PS II and 13 DEGs related to PS I (Fig. 2c). Most of them were up-regulated in both prolonged warming and heat shock (except for $P S B C$, encoding the CP43 subunit of PS II). Particularly, LHCB2.2 and LHCB2.4 (encoding light harvesting complex II), PSB28 (associated with PS II), and PSAH2 and PSAN (related to PS I), were up-regulated by more than 2-fold in prolonged warming than in heat shock (PW vs HS) (Fig. 2c). In 
addition, ATP synthase (ATPC1 and ATPD) in the photosynthetic electron transport system were also up-regulated in prolonged warming vs heat shock, while YMF19 was down-regulated (Fig. 2c).

\section{Respiratory metabolism}

The expression levels of DEGs regulating glyoxylate and dicarboxylate metabolism dramatically increased in prolonged warming vs heat shock. For instance, the expression level of 40-fold higher $M L S$ (encoding malate synthase), more than 8-fold higher RBCS- $1 A, R B C S-1 B, R B C S-2 B$ and $R B C S-3 B$ (encoding ribulose bisphosphate carboxylase), and 3-fold higher HKL1 (encoding hexokinase-like 1), in prolonged warming than in heat shock (Fig. 3a).

By examining the relationship between the expression levels of DEGs and the abundance of metabolites, we identified those pathways that were significantly influenced by both elevated temperature treatments. The major known pathways, including glycolysis, pyruvate metabolism, glyoxylate and dicarboxylate metabolism and TCA cycle, are represented in Fig. 3a. Compared to CK, the metabolism of

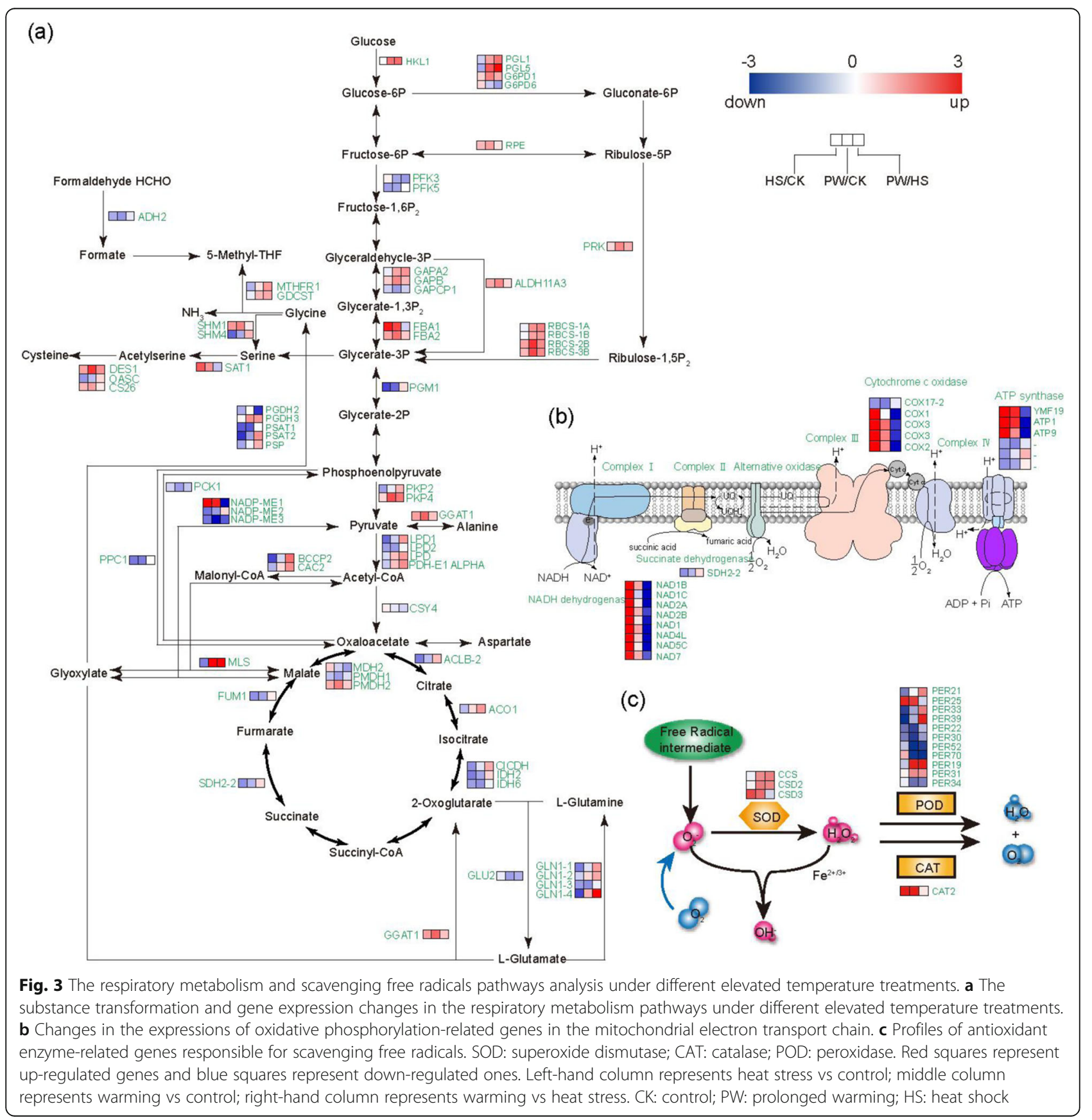


pyruvate genes encoding dihydrolipoyl dehydrogenase (LPD1, LPD2) were down-regulated in heat shock, indicated that heat stress limited the conversion of pyruvate into acetyl-CoA. However, LPD1, LPD and PDH-E1 ALPHA were up-regulated in prolonged warming vs heat shock, indicating a different pattern in this conversion between prolonged warming and heat shock.

In the TCA cycle pathway, genes encoding malate dehydrogenase (PMDH1), fumarate hydratase (FUM1), ATP-citrate synthase beta chain protein $(A C L B-2)$, isocitrate dehydrogenase (IDH2, IDH6) and succinate dehydrogenase $(\mathrm{SDH} 2-2)$ were down-regulated in both prolonged warming and heat shock. This is consistent with the metabolomic results, where citric acid and fumaric acid contents were decreased in prolonged warming vs heat shock (Fig. 2b, Fig. 3a). The decreased malic acid content was also consistent with the downregulation of $P M D H 1$ in prolonged warming vs heat shock (Fig. 2b, Fig. 3a).

In addition, the DEGs related to oxidative phosphorylation, encoding ATP synthase, cytochrome c oxidase and $\mathrm{NADH}$ dehydrogenase, were up-regulated in HS. Examples are ATP synthase protein (YMF19), cytochrome c oxidase subunit (COX1, COX2, COX3), and NADH dehydrogenase (NAD1B NAD1C) (Fig. $3 \mathrm{~b})$. However, most these genes were significantly down-regulated in prolonged warming vs heat shock, indicated that heat shock enhanced more oxidative phosphorylation than prolonged warming.

\section{The antioxidant system}

In SOD-catalyzed reactions, three genes (CCS, CSD2, CSD3) were all up-regulated in prolonged warming. However, only CSD3 was highly expressed in heat shock. Additionally, the expression levels of CCS and CSD2 were 2.5-fold higher in prolonged warming than in heat shock (Fig. 3c).

In CAT-catalyzed reactions, CAT2 (catalase 2) had a higher expression in both heat shock and prolonged warming (Fig. 3c). In addition, most of the genes encoding PODs were down-regulated in heat shock or prolonged warming compared to CK, except for PER25 (peroxidase 25) which had a higher expression in heat shock (Fig. 3c).

\section{Heat shock proteins, transcription factors and heat stress- inducible genes}

The heat shock response network is activated by heat shock proteins thereby initiate heat-stress related transcription factors and genes. Ascorbate peroxidase 2 (APX2) is involved in catalyzing the $\mathrm{H}_{2} \mathrm{O}_{2}$-dependent oxidation of ascorbate in plants. We found that APX2 were only significantly up-regulated in heat shock, but its expression was not detectable in prolonged warming (Fig. 4a). In addition, the expression levels of HSP70-3, HSP70-9, HSP70-14, HSP90-2, HSP90-3 and HSP90-4 were significantly decreased in both prolonged warming and heat shock, and the expression of HSP70-3 was further decreased in heat shock than in prolonged warming (Fig. 4bg). However, HSP22.0 expression was only detected in heat shock, but not in prolonged warming (Fig. 4h).

By going a step upstream, we measured the expression levels of transcription factors including HSFs, DREBs, WRKYs and bZIPs. The expression levels of HSFA1A was slightly up-regulated in heat shock (Fig. $4 \mathrm{i}$ ), but there was no significant difference in HSFA2 expression among $\mathrm{CK}$, prolonged warming and heat shock (Fig. 4j). Interestingly, four $H S F s$, including HSFA6B, HSFA6A, HSFA3 and HSFB2A were significantly up-regulated, and $D R E B 2 A$ and $D R E B 3$ were also strongly up-regulated in heat shock (Fig. 4k). Differently, the expressions of DREB2A and DREB3 decreased in prolonged warming, and most genes encoding WRKY transcription factors were downregulated to a larger extent in heat shock than those in prolonged warming (Fig. 4k). Particularly, five genes encoding bZIPs were significantly up-regulated in prolonged warming but only one (bZIP9) in heat shock (Fig. 4k). The expressions of HSFA4A, HSFA6A, $H S F A 6 B$ and DREB2A detected via qRT-PCR (realtime reverse transcription PCR) showed a similar pattern to those observed in the transcriptome data (Fig. 4l-o), with DREB2C as an exception (Fig. 4p).

We further carried out a series of qRT-PCRs to verify the expression pattern of HSFAs and HSP70/90 in different temperatures with different durations (Fig. 5a-m, Fig. 6a-m, Additional files 5: Table S2). The results showed that in all heat treatments, the HSFA1A, HSFA1B, HSFA2, APX2 and HSP22.0 were significantly upregulated with the increasing of treatment duration (Fig. 5a, b, e, f, g), except that HSFA1D and HSFA1E showed little changes (Fig. 5c, d). However, unlike heat treatments, all HSFA1s and HSFA2 were downregulated as the treated time increasing in all warming treatments (Fig. 6a-e) while APX2 and HSP22.0 were not detected (Fig. 6f, g), indicating that they were largely repressed under prolonged warming. In addition, HSP70/90s were all downregulated with the increasing treating time in both heat shock and prolonged warming (Fig. $5 \mathrm{~h}-\mathrm{m}$, Fig. 6h-m). Among them, under heat shock, HSPS, HSP70-3, HSP70-9, HSP70-14, HSP90-2, HSP90-3 and HSP90-4, exhibited the reverse expression level compared to the HSFA1s.

Most bZIPs were upregulated in prolonged warming on the 7th day, such as bZIP9, bZIP24, bZIP34 and bZIP63 (Fig. 6o, p, q, s). On the other hand, they showed no significantly changes under heat shock (Fig. 5o, p, q, s) except for the downregulated bZIP24 (Fig. 5p), indicating that bZIPs were active in prolonged warming, which were totally different from their expressions in heat shock response. 

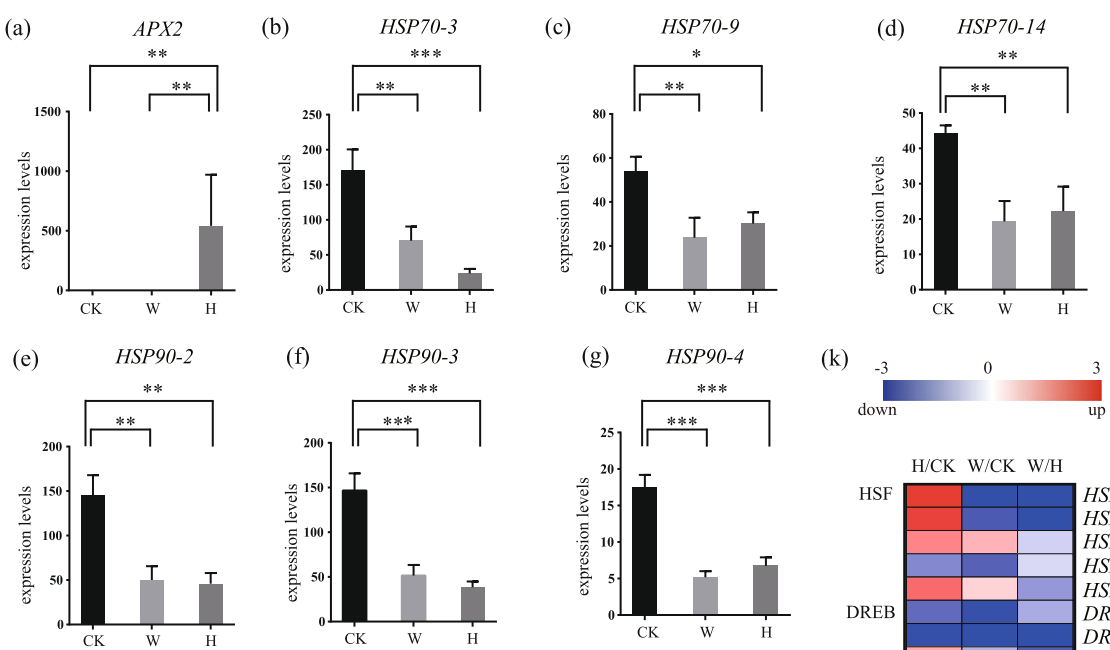

(h)

HSP 22.0

(i)

HSFA1A

(j)

HSFA2
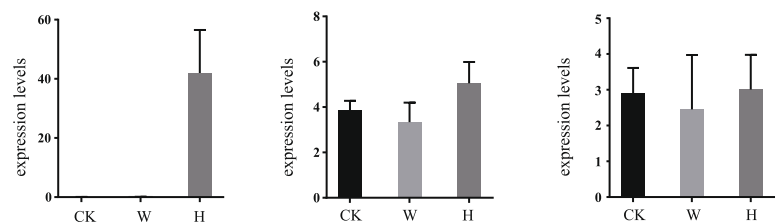

(1)

(m)

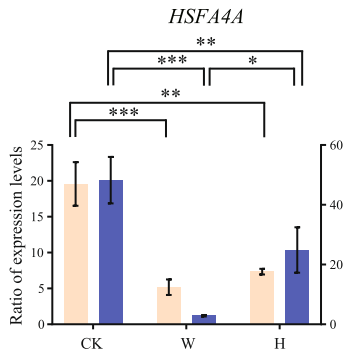

HSFA6A
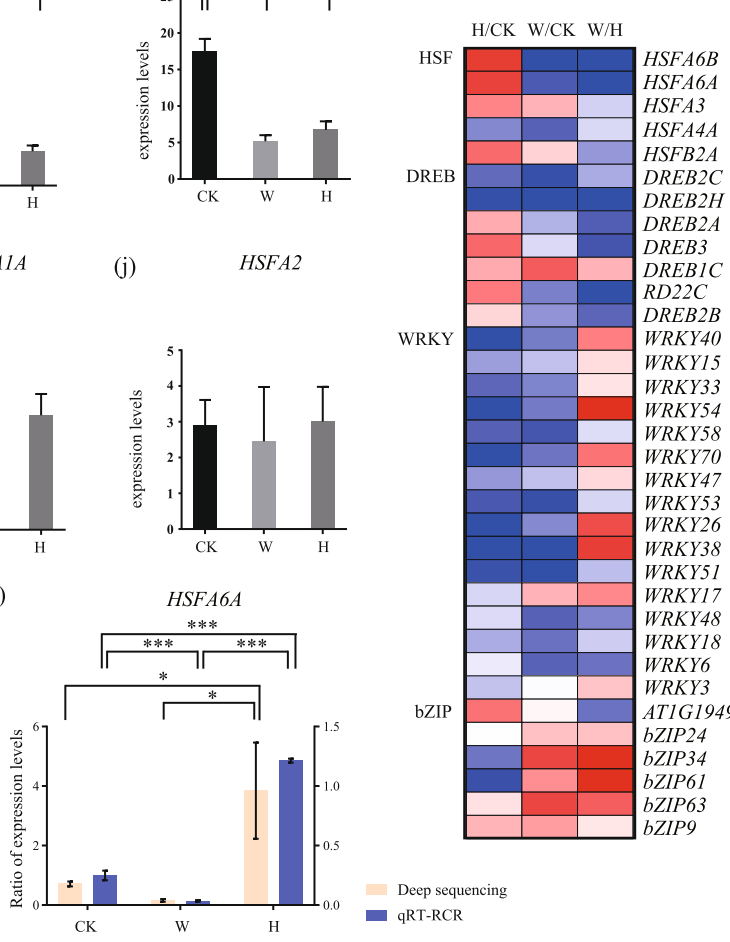

WRKY

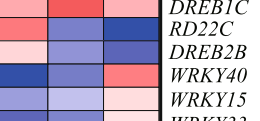

(o)

DREB2A

(p)
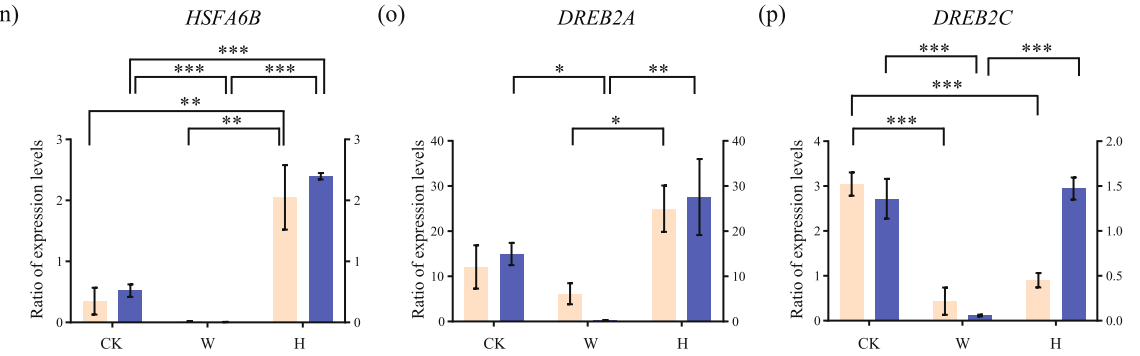

Fig. 4 Heat shock protein and transcription factor analyses under different elevated temperature treatments. Expression levels of (a) ascorbate peroxidase 2 (APX2), (b) heat shock protein 70-3 (HSP70-3), (c) HSP70-9, (d) HSP70-14, (e) HSP90-2, (f) HSP90-3, (g) HSP90-4, (h) HSP22.0, (i) heat shock factor A 1A (HSFA1A), and (j) HSFA2. Each bar represents mean \pm SD; $n=3$. $\mathbf{k}$ Heatmap of 34 differentially expressed transcription factors under control, prolonged warming and heat shock treatments, grouped into 4 main categories. Red rectangles mean up-regulation of expression and blue means down-regulation. I-p The expression levels of several selected transcription factors: (I) HSFA4A, (m) HSFA6A, (n) HSFA6B, (o) $D R E B 2 A$, and (p) DREB2C were determined using quantitative RT-PCR analyses. Pink bars: results from deep sequencing; blue bars: results from qRT-PCR. Each bar represents mean \pm SD; $n=3$. CK: control; PW: prolonged warming; HS: heat shock

\section{Discussion}

By systematic comparison of the physiological, transcriptional, and metabolic responses of Arabidopsis leaves toward prolonged warming and heat shock, we revealed the differential response patterns between these two types of heat stresses, which involve multiple components including photosynthesis, respiration, ROS scavenging, and stress signaling pathways. 


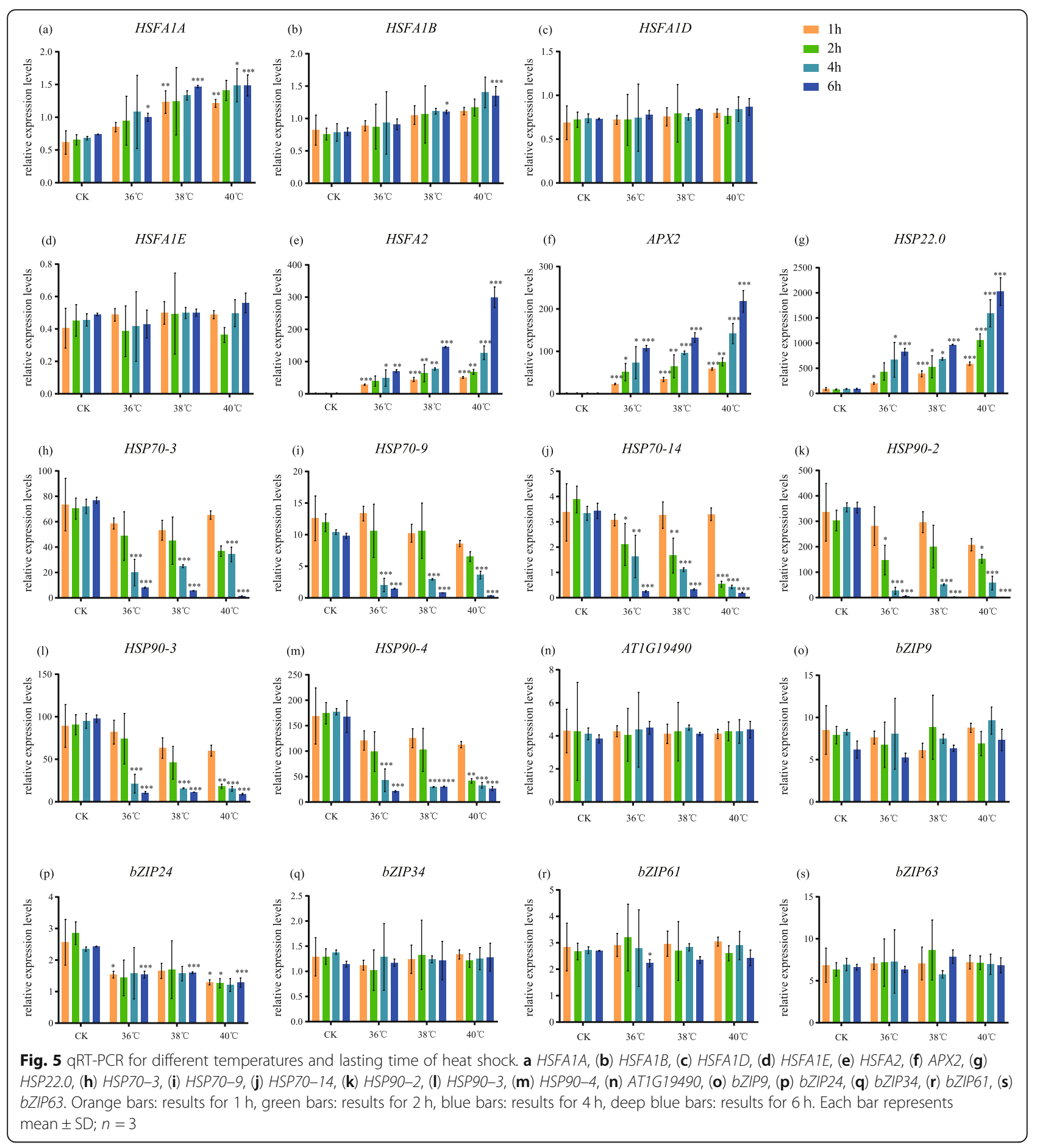

To determine the gas exchange under prolonged warming and heat shock conditions, we measured the stomatal conductance. We found that with prolonged warming treatment, the stomatal conductance decreased significantly, and the rate of $\mathrm{CO}_{2}$ assimilation was also significantly inhibited. However, they remained largely unchanged under heat shock which led to high transpiration rate. Stomatal regulation is a vital protective mechanism for high-temperature tolerance as it is crucial for desiccation prevention. Generally, under moderate to severe stress, the photosynthetic rate would decrease due to lowered mesophyll conductance and stomatal closure [12]. Therefore, our results suggest two different response mechanisms. Under heat shock, because plants experience a sudden increase in temperature, they may increase the rate of transpiration to allow the cooling effect due to 


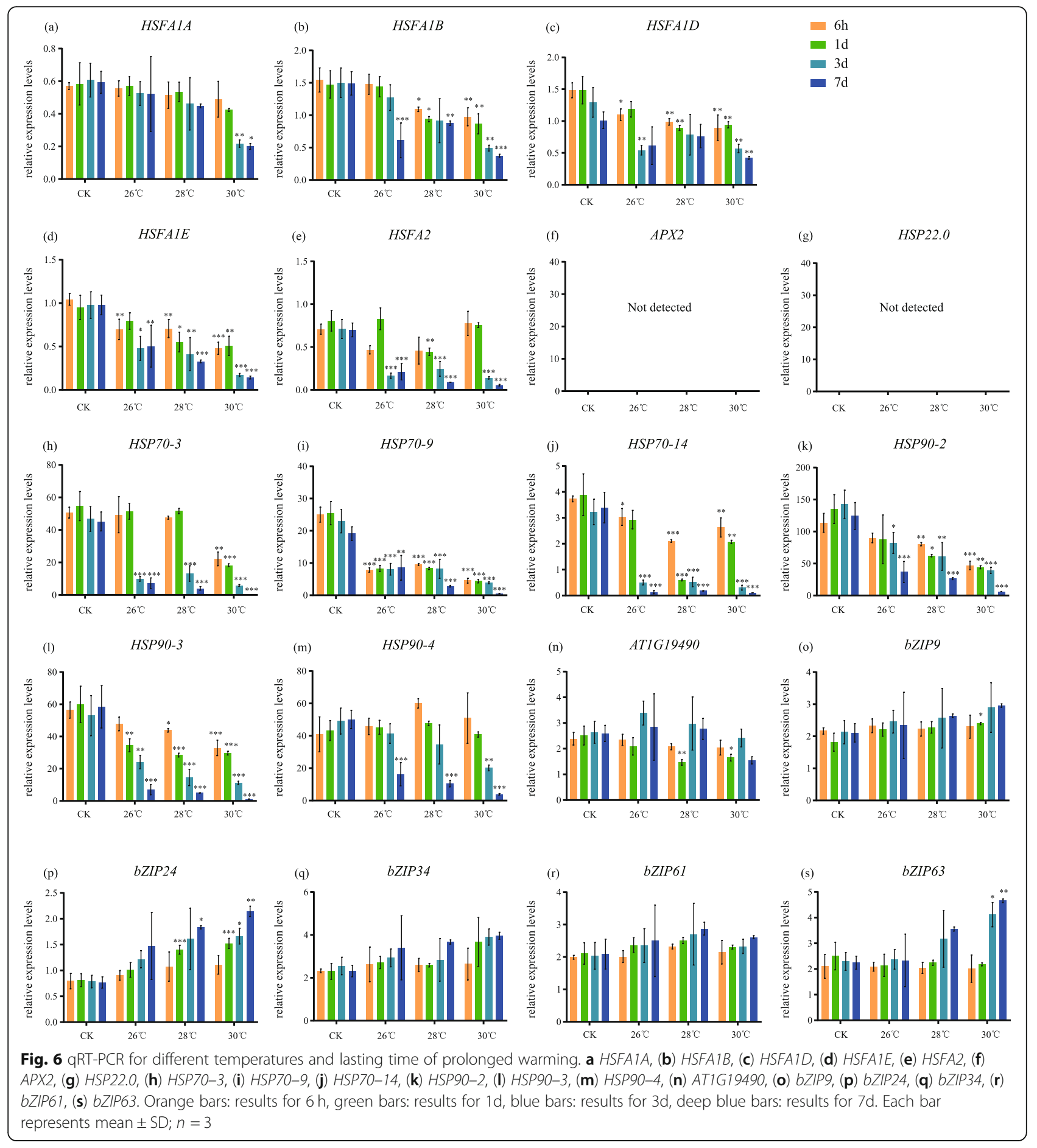

evaporation. Differently, under prolonged warming in which the temperature increase is not lethal, the plants close their stomata to prevent excessive water loss.

We further investigated the responses of photosynthesis under prolonged warming and heat shock, and revealed that photochemical quenching decreased and non-photochemical quenching increased under prolonged warming, while the genes related to LHCII and the photosynthetic electron transport system including PSII and PSI, such as LHCB2.2, LHCB2.4, PSB28, $P S A H 2, P S A N$, were up-regulated. Previous studies have identified that some PSII-related protein subunits and cofactors of the photosynthetic electron transport system responsive to high temperature. Psb28-1 plays an important role in PSII repair at high temperatures $[16,17]$. LHCII, as the major component of PSII, functions in 
light energy distribution and light protection, and Lhcb1 and Lhcb2 are primary constituents of mobile trimeric LHCIIs [18]. Combining with these studies, we predicted that the enhanced photoreaction and photoprotection under prolonged warming condition. Similarly, most genes related to light harvesting complexes and the photosynthetic electron transport system were also upregulated with heat shock, suggesting that short-term high temperature may also induce photoprotection.

Respiration usually involves pathways of the glycolysis, TCA cycle, mitochondrial electron transport chain (miETC) and oxidative phosphorylation. Elevated temperatures can induce damages in the plant cell by upsetting the balance in cellular respiration [1]. Our data showed that the glycolysis pathway-related genes, including HKL1, GAPA2, GAPB, FBA1, FBA2 and PKP4, were up-regulated with prolonged warming treatment. Meanwhile, the genes associated with the TCA cycle, such as FUM1, PMDH1 and ACLB-2, were downregulated, and the metabolomics results confirmed the decrease in TCA cycle activity. These results suggested that some specific TCA cycle intermediates were highly depleted by prolonged warming conditions. On the contrary, under heat shock, some glycolysis pathway-related genes, such as PKP2, LPD1 and LPD2 were downregulated, while some genes related to the respiratory electron transfer and oxidative phosphorylation pathways, such as NAD1B, NAD1C, COX1, COX2, and $C O X 3$, were significantly induced, indicating that heat stress inhibited the glycolysis and TCA cycle pathways while enhancing electron transport.

Soluble carbohydrates and amino acids (such as proline) are important primary metabolites related to heat stress in plants, which were synthesized from the intermidiate metabolites from glycolysis and TCA cycle. The accumulation of soluble sugars, which are associated with cellular osmotic homeostasis and membrane stability, could protect the photosynthetic apparatus from heat damage and maintain photosynthetic capacity $[19,20]$. In this study, soluble sugars were significantly increased under both prolonged warming and heat shock. Compared to prolonged warming, heat shock resulted in markedly higher concentrations of soluble sugars. In addition, the patterns of sugar alcohol and carbohydrate conjugate accumulation in response to the two treatments were quite different. For example, sorbitol accumulated extensively only with prolonged warming but not under heat shock, while galactonic acid, mannose, methyl-beta-D-galactopyranoside, and phenyl-beta-D-glucopyranoside were significantly reduced under heat shock. Since the osmotic substances were produced through photosynthetic assimilates or respiratory intermediate products, the ATP and NADPH were needed as the reducing power provider, which is mainly generated from respiration. Our results suggest that the molecules needed for maintaining osmotic balance during prolonged warming and heat shock might have been produced through the intermediate products of glycolysis.

Under abiotic stresses, plants usually accumulate ROS. At the same time, plants have expeditious antioxidant systems, including non-enzymatic antioxidants such as ascorbate (ASC) and glutathione (GSH), as well as antioxidant enzymes such as SOD, POD and CAT responsible for ROS scavenging and removal. However, once the equilibrium between the generation and scavenging of ROS is disrupted under stress conditions, ROS start to accumulate $[21,22]$. Here we found that the concentration of $\mathrm{H}_{2} \mathrm{O}_{2}$, the activities of SOD and CAT, and their related genes, including CSD3 and CAT2, were all enhanced under both prolonged warming and heat shock treatments, indicating that both types of treatments can induce ROS-scavenging enzyme activities to detoxify ROS. However, the concentration of $\mathrm{H}_{2} \mathrm{O}_{2}$ increased more dramatically upon rapid heat shock. Moreover, POD activity was higher under heat shock than with prolonged warming, and POD synthesis-related gene PER25 expression was significantly up-regulated under heat shock vs prolonged warming, indicating the significant accumulation of ROS, and the disruption of the equilibrium between ROS generation and scavenging systems under heat shock. With prolonged warming, the level of ROS, although elevated from the control level, was still relatively low. Actually, several lines of evidence have shown that when under moderate stress, the scavenging system could keep the ROS level low, with ROS serving as signaling molecules which activate an acclimation response and programmed cell death. For example, at moderate stress, ROS play a crucial role in intracellular signaling from the chloroplast to the nucleus to control plant development processes [23]. Therefore, at this point, we postulate that ROS might function as signaling molecules to regulate the activation of stress response pathways, and did not result in the irreversible inactivation of the photosynthetic system or cause grave damage to PSII under prolonged warming. However, the detailed mechanisms await further investigation.

In anticipation of upcoming damaging conditions, plants can activate genes and accumulate HSPs involved in cellular defenses against heat damage. HSPs, including HSP100, HSP90, HSP70, HSP60, and small HSPs, have critical roles in regulating protein quality by renaturing a variety of proteins denatured due to heat stress. These HSPs are in turn precisely controlled by a network of transcription factors (TFs), including HSFs, DREBs, WRKYs, and bZIPs [24]. Recent reviews have elucidated the complex transcriptional and post-translational regulatory networks involved in heat stress [13, 25]. HSFs are the terminal components of a signal transduction chain mediating 
the activation of genes responsive to heat stress, which are particularly important in thermotolerance responses [13]. In this study, transcriptome analysis by RNA-seq detected 33 TF families, including HSFs, DREBs, WRKYs, and bZIPs that responded to heat stress. Among them, the transcription factors in HSFA1s and DREBs pathways, such as HSFA1A, HSFA6A, HSFA6B and DREB2A were up-regulated in heat shock treatment. In Arabidopsis, HSFA1s were shown to play a central role in the heat stress response. Many important heat stress response TFs, such as DREB2A, HSFA2, HSFA7a, and HSFBs have been predicted to be directly regulated by HSFA1s [26]. Therefore, our data suggest that the HSFA1s and DREBs play a crucial role in response to heat shock. HSP70 and HSP90 can repress HSFA1 activity through the repression of its transactivation activity and nuclear localization, respectively. Upon heat shock, HSFA1s are dissociated from HSP70 and derepressed [13]. Similarly, our qRT-PCR results showed that all HSP70/90s were significantly down-regulated and their corresponding HSFA1s were up-regulated in heat shock. These results further confirmed that HSFA1 becomes active from the repression of HSP70/ 90, and HSFA1s are negatively regulated by HSP70/ 90. However, in prolonged warming treatments, although the HSP70/90s showed low expression levels, the HSFA1s were downregulated, suggesting that HSFA1s did not act as the central regulator in response to prolonged warming.

bZIP TFs are endoplasmic reticulum stress sensors in plants, which regulate many processes including abscisic acid (ABA) and stress signaling, and contribute to stress tolerance [27]. In our study, it is interesting to point out that, with prolonged warming, the transcription factors in an HSFA1-independent pathway, such as bZIP9, bZIP24, bZIP34 and bZIP63 were up-regulated. In addition, our large scale qRT-PCR results also validated that these bZIP family members, such as bZIP24 and bZIP34, were up-regulated in all prolonged warming treatments (e.g., the 7th day), whereas none of them were upregulated in heat shock. These results suggest that prolonged warming and heat shock may induce entirely different heat response pathways for thermotolerance or thermo-acclimation.

Besides, early exposure to a mild temperature stress can enhance thermotolerance to heat stress, and the stress priming may occur at the level of gene transcription, such as HSFA2 expression depending on the expressed HSFA1 isoforms [28, 29]. Additionally, HSP22.0 and APX2 associated with heat stress priming can remain elevated levels for several days in heat memory [28]. Here, we found that HSFA2, HSP22.0 and $A P X 2$ were only up-regulated under heat shock but not with prolonged warming, indicating that prolonged warming treatment conditions $\left(5^{\circ} \mathrm{C}\right.$ above control) may not be enough to prime the plant to subsequently withstand high temperatures in Arabidopsis.

\section{Conclusions}

Our research provided detailed information on the physiological, transcriptional, and metabolic responses of Arabidopsis to prolonged warming versus heat shock (summarized in Fig. 7). On the basis of these multi-level results, we conclude that plants respond to rapid-onset heat shock mainly through increasing the rate of transpiration, the rate of photosynthetic and respiratory electron transfer, the production of ROS, the induction of antioxidant enzymes, and the activation of the HSFA1 heat stress response pathway. On the other hand, plants

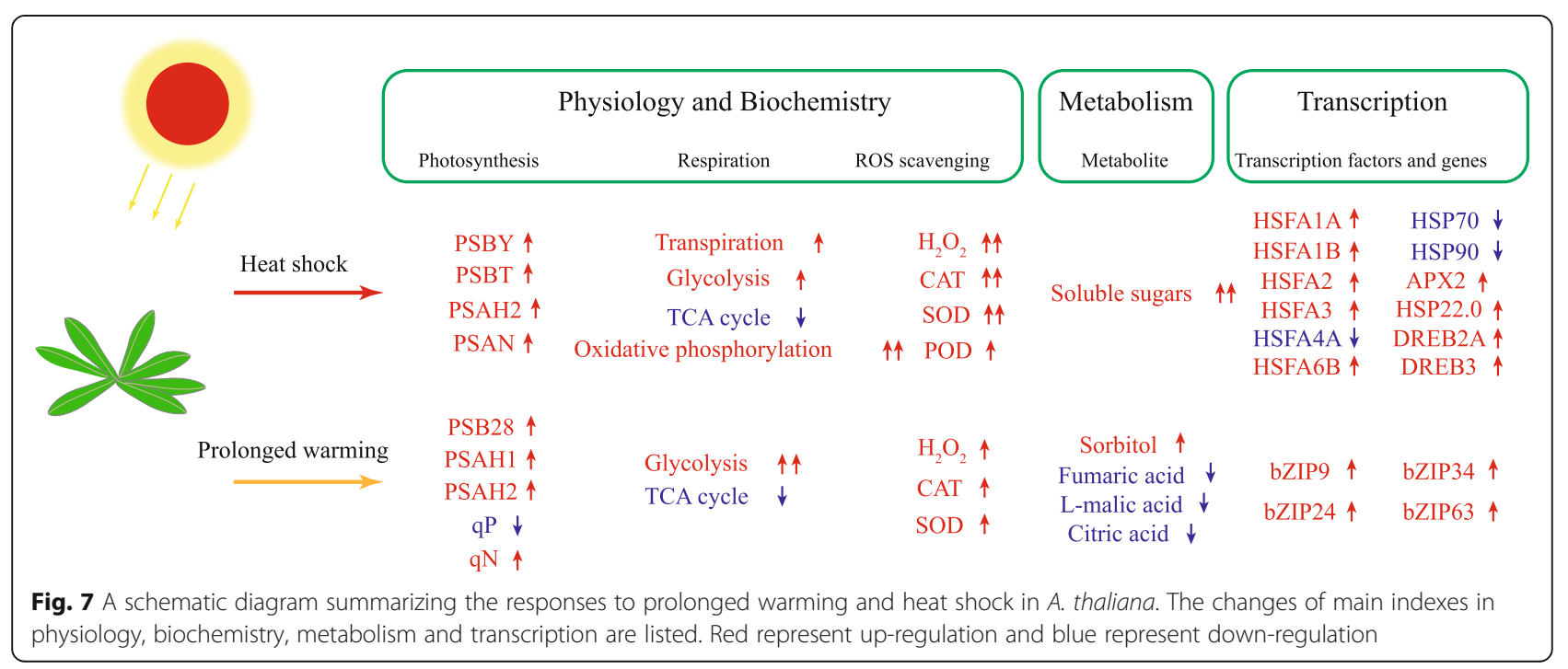


respond to prolonged warming primarily via decreased stomatal conductance, increased photosynthetic electron transfer rate, inhibited TCA cycle, and activation of a HSFA1-independent response pathway of bZIPs.

\section{Methods}

\section{Plant materials and growth conditions}

The seeds of wild-type (WT) Arabidopsis thaliana Columbia ecotype (Col-0) were originally acquired from Nottingham Arabidopsis Stock Centre, Nottingham University, UK, and have grown in growth chambers at 23/ $18^{\circ} \mathrm{C}$ (day/night) for more than 30 generations by seed propagation over the past 10 years in our laboratory. Seeds were stratified at $4{ }^{\circ} \mathrm{C}$ for 2 days, and then sown in pots $(650 \times 650 \times 750 \mathrm{~mm})$ filled with a mixture of vermiculite and peat $(1: 1, \mathrm{v} / \mathrm{v})$, and placed in growth chambers (RXZ-300B, NingboDongnan Instruments Co Ltd., China) under $23^{\circ} \mathrm{C}(16 \mathrm{~h}) / 18^{\circ} \mathrm{C}(8 \mathrm{~h})$ (day/night) with day light intensity $350 \mu \mathrm{mol} \cdot \mathrm{m}^{-2} \cdot \mathrm{s}^{-1}$. Relative humidity (RH) was maintained at $80 \% / 90 \%$ (day/night). After true leaf emergence, seedlings were thinned to two or three plants per pot and the pots were randomly rearranged every 3 days to offset position effects within the chambers (Fig. 1a). The plants were alternately watered with 1/2 Murashige and Skoog solution or with de-ionized water once a week. To eliminate possible differences among the different growth chambers, plants of each treatment were exchanged and relocated in different growth chambers $(\geq 3)$ irregularly during plant growth and treatment. Besides, although the sensitivity of the growth chamber is in the range of $\pm 0.5^{\circ} \mathrm{C}$, two thermometers were placed in each growth chamber for checking the accuracy of the setting temperatures.

\section{Control}

The day/night temperatures were set at $23 / 18^{\circ} \mathrm{C}$ as the control temperature (CK) based on published studies using the Col-0 ecotype $[8,30]$. Leaves were sampled from plants at 30 days after sowing (at the rosette growth stage) (Fig. 1b).

\section{Prolonged warming treatment}

The global mean temperature is likely to warm by $1.5-$ $4{ }^{\circ} \mathrm{C}$ at the end of this century [31]. Therefore, we set the warming temperature at $5^{\circ} \mathrm{C}$ above $\mathrm{CK}$, and plants at 23 days after sowing were subjected to $28 / 23^{\circ} \mathrm{C}$ (day/ night) for 7 days as the prolonged warming treatment. After warming treatment, leaves were sampled for analyses (Fig. 1b).

\section{Heat shock treatment}

Since $37-42^{\circ} \mathrm{C}\left(44-45^{\circ} \mathrm{C}\right.$ being the lethal temperature) had been widely used as the temperature in Arabidopsis heat stress studies, we set $38^{\circ} \mathrm{C}$ for $6 \mathrm{~h}$ during the day portion of the photoperiod as the heat shock treatment (Fig. 1b).

Hence, all leaf sampling in the three temperature regimes were done with plants at 30 days after sowing (growth stage 3.90). At this stage, the plant rosette growth is nearly complete and leaves are fully expanded [32]. All leaf samples were immediately snap-frozen in liquid nitrogen. The same batch of sampling materials were used for transcriptome, metabolome, physiological and biochemical analyses.

\section{RNA extraction, RNA sequencing and data analyses}

Total RNA was isolated separately from the leaves subjected to control, prolonged warming and heat shock treatments, respectively, with three biological replicates each, for RNA extraction and sequencing. All total RNA samples were extracted using the Mini BEST Plant RNA Extraction Kit (TaKaRa, Dalian, China) and treated with genomic DNA Eraser (TaKaRa, Dalian, China) to reduce or eliminate any DNA contamination. Illumina-based RNA sequencing was performed on the Hiseq ${ }^{\mathrm{Tm}} 4000$ platform. After removing the reads containing adapter, reads containing ploy- $\mathrm{N}$ and low quality reads, the filtered reads were mapped to the A. thaliana genome (TAIR 10) using TopHat2. Then Reads Per Kilobase of transcript per Million mapped reads (RPKM) of each gene was calculated based on the length of the gene and reads count mapped to this gene. Differential expression analysis among the samples was performed using the DESeq $R$ package (1.18.0). Thresholds of $\mid \log _{2}$ (fold change) $\mid \geq 1$ and adjusted $P$-value $<0.05$ were applied to assess the significance of the differences in transcript levels. The sequencing data have been deposited in the NCBI Gene Expression Omnibus (GEO) database under the accession number GSE118298.

\section{Metabolome analysis}

A volume of $0.48 \mathrm{~mL}$ methanol-water $(3: 1, \mathrm{v} / \mathrm{v})$ and $24 \mu \mathrm{L}$ of adonitol $\left(1 \mathrm{mg} / \mathrm{mL}\right.$ stock in $\left.\mathrm{dH}_{2} \mathrm{O}\right)$ were added to $0.06 \mathrm{~g}$ of each sample in a $2 \mathrm{~mL}$ Eppendorf tube as internal standard, followed by homogenization in a ball mill for $4 \mathrm{~min}$ at $50 \mathrm{~Hz}$, and then sonication for $5 \mathrm{~min}$ twice (with incubation in ice water). After centrifugation at $13000 \times \mathrm{g}$ at $4{ }^{\circ} \mathrm{C}$ for $15 \mathrm{~min}, 350 \mu \mathrm{L}$ of supernatant was transferred into a fresh $2 \mathrm{~mL}$ GC/MS glass vial. After drying the samples with a vacuum concentrator, $80 \mu \mathrm{L}$ methoxyamine hydrochloride $(20 \mathrm{mg} / \mathrm{mL}$ in pyridine) was added to each sample and incubated at $80^{\circ} \mathrm{C}$ for 30 min. Then, $100 \mu \mathrm{L}$ of the BSTFA regent (1\% TMCS, v/v) was added and the mixture incubated at $70^{\circ} \mathrm{C}$ for $1.5 \mathrm{~h}$ and mixed well for GC-time-of-flight (TOF)-MS analysis.

GC-TOF-MS analysis was performed using an Agilent 7890 gas chromatograph system coupled with a Pegasus 
HT time-of-flight mass spectrometer (Agilent Technologies, Santa Clara, CA, USA). Each treatment in this metabolomics study was repeated with at least six biological replicates.

Chroma TOF 4.3X software of LECO Corporation and LECO-Fiehn Rtx 5 database were used for raw peaks exacting, data baselines filtering, baseline calibration, peak alignment, deconvolution analysis, peak identification and integration of the peak area. The RI (retention time index) method was used for peak identification, with the RI tolerance at 5000 .

\section{Measurement of photosynthetic capacity}

The main photosynthetic parameters were measured on mature leaves using a Portable Photosynthesis System (LI-6400XT) to quantify $\mathrm{CO}_{2}$ uptake under conditions of saturating light and water availability. All samples were measured on intact plants in the growth chambers under the three different treatments. During all measurements, a high flow rate $\left(400 \mathrm{~mL} \cdot \mathrm{min}^{-1}\right)$ through the cuvette was maintained to keep the $\mathrm{CO}_{2}$ concentration within the range of $370-390 \mu \mathrm{mol} \cdot \mathrm{mol}^{-1}$. The temperature in the leaf chamber was kept the same as the treatment temperature and all measurements were carried out between the eighth and ninth hour of daylight in the growth chambers. Light intensities of $1000 \mu \mathrm{mol}$ quanta $\mathrm{m}^{-2} \cdot \mathrm{s}^{-1}$ were used in the Photosynthesis System as the saturating photosynthetic photon flux density of Arabidopsis. Eight leaves from eight different plants were measured to provide biological replicates in each treatment. All the data collected at steadystate after inserting leaves into the leaf chamber.

\section{$\mathrm{H}_{2} \mathrm{O}_{2}$ level analysis}

Freshly cut leaf samples $(0.1 \mathrm{~g})$ were homogenized in an ice bath with $0.9 \mathrm{~mL}$ of $50 \mathrm{mmol} \cdot \mathrm{L}^{-1}$ phosphate buffer (PH7.8) and centrifuged at $10,000 \times \mathrm{g}$ for $10 \mathrm{~min}$ at $4{ }^{\circ} \mathrm{C}$. The $\mathrm{H}_{2} \mathrm{O}_{2}$ concentration in the supernatant was determined by a colorimetric method using a commercial kit (BCA assay, Nanjing Jiancheng Bioengineering Institute, Nanjing, China). Three to five leaves were used to provide enough amount of leaf tissues for each sample (three biological replicates per treatment).

\section{Physiological Indicator measurements}

The soluble sugar concentration in the supernatant was determined by anthrone colorimetry, and the soluble protein concentration in the supernatant was determined by the Coomassie Brilliant Blue method [33]. Freshly cut leaf samples $(0.1 \mathrm{~g})$ were homogenized in an ice bath with $1 \mathrm{~mL}$ of distilled water and put into a water bath at $95^{\circ} \mathrm{C}$ for $10 \mathrm{~min}$. After cooling, the samples were centrifuged at $8000 \times \mathrm{g}$ for $10 \mathrm{~min}$ at $25^{\circ} \mathrm{C}$, and diluted with distilled water to $10 \mathrm{~mL}$. Three to five leaves were used to provide enough amounts of leaf tissues for each replicate (three biological replicates per treatment).

The superoxide radical scavenging ability in the supernatant was determined using a commercial kit (BCA assay, Nanjing Jiancheng Bioengineering Institute, Nanjing, China). The activities of three enzymes SOD, CAT and POD were determined using commercial kits (Suzhou Comin Bioengineering, Suzhou, China). Three to five leaves were used to provide enough amounts of leaf tissues for each replicate (three biological replicates per treatment).

\section{Real-time reverse transcription PCR (qRT-PCR) analysis}

We performed qRT-PCR to verify the results of transcriptome. In addition, to validate the expression pattern of some selected genes encoding important functions such as transcription factors, we performed a series of qRT-PCRs in different temperatures with different durations. For heat shock, we included treatments at $36^{\circ} \mathrm{C}, 38^{\circ} \mathrm{C}$ and $40^{\circ} \mathrm{C}$, and sampled the leaves at $1 \mathrm{~h}, 2 \mathrm{~h}, 3 \mathrm{~h}$ and $6 \mathrm{~h}$ after treatments. For prolonged warming, we included treatments at $26^{\circ} \mathrm{C}, 28^{\circ} \mathrm{C}$ and $30^{\circ} \mathrm{C}$ and sampled the leaves at $6 \mathrm{~h}, 1 \mathrm{~d}$, $3 \mathrm{~d}$ and $7 \mathrm{~d}$ after treatments. All leaf samples were immediately snap-frozen in liquid nitrogen.

Each RNA sample (containing about $1 \mu \mathrm{g}$ of total RNA) was treated with gDNA Eraser (TaKaRa, Dalian, China) following the manufacturer's instructions, to eliminate any contaminant gDNA. The treated RNA solution $(10 \mu \mathrm{L})$ was subjected to reverse transcriptase reactions with PrimeScript ${ }^{\mathrm{tm}}$ Reverse Transcriptase Reagent Kit with gDNA Eraser (Perfect Real Time) (TaKaRa, Dalian, China) in accordance with the manufacturer's protocol. Gene-specific primers were designed using Primer 5.0. Actin2 mRNA was used as the internal reference gene. Quantitative RT-PCR was performed using a Bio-Rad CFX96 ${ }^{\text {TM }}$ Real-Time System (Bio-Rad, USA) using the SYBR Premix Ex Taq ${ }^{\text {TM }}$ Kit (Perfect Real Time) (TaKaRa, Japan) in accordance with the manufacturer's protocol. qRT-PCR conditions were as follows: $30 \mathrm{~s}$ at $94{ }^{\circ} \mathrm{C}$ for denaturation, 40 cycles for $5 \mathrm{~s}$ at $94{ }^{\circ} \mathrm{C}, 30 \mathrm{~s}$ at $56^{\circ} \mathrm{C}$, and $10 \mathrm{~s}$ at $72{ }^{\circ} \mathrm{C}$. Relative expression levels of target genes were calculated with the $2^{-\Delta \Delta \mathrm{Ct}}$ comparative threshold cycle (Ct) method. All reactions were performed in three biological replicates, and the results of $\mathrm{Ct}$ values were determined with Bio-Rad CFX Manager V1.6.541.1028 software.

\section{Statistical analysis}

Statistical significance of differences in this study was analyzed using one-way ANOVA followed by Tukey's post hoc test with a significance level of $0.05(p<0.05)$ (SPSS 18.0 software for Windows) (SPSS, Chicago, IL, USA) [34]. 


\section{Supplementary information}

Supplementary information accompanies this paper at https://doi.org/10. 1186/s12870-020-2292-y.

Additional files 1: Figure S1. Principal component analysis (PCA) and Orthogonal projection to latent structure with discriminant analysis (OPLS-DA) of Metabolomic analyses. (a) PCA scores plot. (b)-(d) OPLS-DA scores: (b) control (CK) vs heat shock (HS); (c) control (CK) vs prolonged warming (PW); (d) prolonged warming (PW) vs heat shock (HS). Black circle: control; red square: prolonged warming; blue triangle: heat shock. Each treatment contains six biological repeats.

Additional file 2: Figure S2. Gene expression comparisons. (a) Venn diagram of the number of differentially expressed genes (DEGs). Numbers in the overlapping sets show the number of genes that are differentially expression in two or three pairwise comparisons. (b)-(d) Kyoto Encyclopedia of Genes and Genomes (KEGG) pathway enrichment of DEGs. (b) Control (CK) vs heat shock (HS); (c) control (CK) vs prolonged warming (PW); (d) prolonged warming (PW) vs heat shock (HS). The sizes of the dots are in proportion to the number of genes involved. The color of the dot is closer to red as the q-value approaches 0 . The genes are considered statisticallysignificantly over-represented, i.e. enriched, when $q<0.05$. Red arrows in (b) and (c) indicate those pathway enrichment of DEGs involved in ribosome, antenna proteins, photosynthesis and citrate cycle; Red arrows in (d) indicate the most siginificant enriched pathways.

Additional file 3: Figure S3. Gene ontology (GO) term enrichment of DEGs. (a) Heat shock (HS) vs control (CK); (b) prolonged warming (PW) vs control (CK); (c) prolonged warming (PW) vs heat shock (HS) Green bars represent genes involved in biological processes in response to external stimuli, and orange bars represent those involved in the structures of cellular components.

Additional file 4: Table S1. Summary of draft reads of samples by Illumina deep sequencing.

Additional file 5: Table S2. Primers used in qRT-PCR of the leaves of $A$ thaliana.

\section{Abbreviations}

ABA: Abscisic acid; ACLB: ATP-citrate synthase beta chain; APX2: Ascorbate peroxidase 2; ASC: Ascorbate; bZIP: Basic leucine zipper; CAT: Catalase; CAT2: Catalase 2; CCS: Copper chaperone for superoxide dismutase; Chl: Chlorophyll; CK: Control; COX 1: Cytochrome c oxidase subunit 1; CSD: Superoxide dismutase; Cyt: Cytochrome b6f complex;

DEGs: Differentially expressed genes; DREBs: Dehydration responsive element-binding protein; FBA1/2: Fructose-bisphosphate aldolase 1/2; Fdx: Ferredoxin; FUM1: Fumarate hydratase; GAPA2: Glyceraldehyde 3phosphate dehydrogenase A subunit 2; GAPB: Glyceraldehyde-3-phosphate dehydrogenase B subunit; GEO: Gene Expression Omnibus; GO: Gene ontology; GSH: Glutathione; $\mathrm{H}_{2} \mathrm{O}_{2}$ : Hydrogen peroxide; HKL 1: Hexokinase-like 1; HS: Heat shock; HSF: Heat shock factor; HSFA1: Class A1 heat shock factor; HSPs: Heat shock proteins; IDH: Isocitrate dehydrogenase; KEGG: Kyoto Encyclopedia of Genes and Genomes; LHC I/II: Light-harvesting complex I/II; LPD: Dihydrolipoyl dehydrogenase; miETC: Mitochondrial electron transport chain; MLS: Malate synthase; NAD1B/C: NADH dehydrogenase; NADH: Nicotinamide adenine dinucleotide; OPLS-DA: Orthogonal projection to latent structure with discriminant analysis; PC: Plastocyanin; PCA: Principal component analysis; PER25: Peroxidase 25; PKP4: Plastidial pyruvate kinase 4; PMDH1: Protein malate dehydrogenase 1; POD: Peroxidase; PQ: Plastoquinone; PS I/II: Photosystem I/II; PW: Prolonged warming; qN: Non-photochemical quenching; qP: Photochemical quenching; qRTPCR: Real-time reverse transcription PCR; RBCS: Ribulose bisphosphate carboxylase; ROS: Reactive oxygen species; SDH: Succinate dehydrogenase; SEM: Scanning electron microscopy; SOD: Superoxide dismutase; TCA: The tricarboxylic acid; TFs: Transcription factors; WRKYs: WRKY transcription factors; YMF19: ATP synthase protein YMF19

\section{Acknowledgements}

We thank Novogene Company (Beijing) for helping with transcriptome sequencing and Biotree Bio-technology (Shanghai) for helping with metabolomics data analysis. We thank Dr. LX Huang-Fu for the assistance in measuring the photosynthetic capacity. JY Chu copy-edited the manuscript.

\section{Authors' contributions}

LW, KBM, HML and BJ designed the research. KBM, ZGL and SXR performed the experiments and analysed results. $L W, K B M, B J$ and $H M L$ wrote the manuscript. HRJ, JWC, GC and NJT participated to the result analyses and interpretation of data. HML revised the manuscript critically. All authors have read and approved the final manuscript.

\section{Funding}

This work was funded by the National Key Research and Development Program of China (2016YFD0600105) awarded to NJT and the Hong Kong Research Grants Council Area of Excellence Scheme (AoE/M-403/16) awarded to HML. The funder has no role in the design of the study and collection, analysis, and interpretation of data and in writing the manuscript.

\section{Availability of data and materials}

The sequencing data are available in the NCBI Gene Expression Omnibus (GEO) database under the accession number GSE118298. The datasets supporting the results of this article are included within the article and the additional files.

\section{Ethics approval and consent to participate}

Not applicable.

\section{Consent for publication}

Not applicable.

\section{Competing interests}

The authors declare that they have no competing interests.

\section{Author details}

${ }^{1}$ College of Horticulture and Plant Protection, Yangzhou University, Yangzhou 225009, China. ${ }^{2}$ Center for Soybean Research of the State Key Laboratory of Agrobiotechnology and School of Life Sciences, The Chinese University of Hong Kong, Hong Kong, SAR, China. ${ }^{3}$ College of Bioscience and Biotechnology, Yangzhou University, Yangzhou 225009, China. ${ }^{4}$ College of Horticulture, Nanjing Agricultural University, Nanjing 210095, China.

Received: 10 March 2019 Accepted: 14 February 2020

Published online: 22 February 2020

\section{References}

1. Wahid A, Gelani S, Ashraf M, Foolad MR. Heat tolerance in plants: an overview. Environ Exp Bot. 2007;61:199-223. https://doi.org/10.1016/j. envexpbot.2007.05.011.

2. Wolkovich EM, Cook BI, Allen JM, Crimmins TM, Betancourt JL, Travers SE, et al. Warming experiments underpredict plant phenological responses to climate change. Nature. 2012:485:494-7. https://doi.org/10.1038/ nature11014.

3. Springate DA, Kover PX. Plant responses to elevated temperatures: a field study on phenological sensitivity and fitness responses to simulated climate warming. Glob Chang Biol. 2014;20:456-65. https://doi.org/10.1111/gcb. 12430.

4. Hedhly A, Hormaza Jl, Herrero M. Global warming and sexual plant reproduction. Trends Plant Sci. 2009;14:30-6. https://doi.org/10.1016/j. tplants.2008.11.001.

5. Ainsworth EA, Ort DR. How do we improve crop production in a warming world? Plant Physiol. 2010;154:526-30. https://doi.org/10.1104/pp.110. 161349.

6. Lin D, Xia J, Wan S. Climate warming and biomass accumulation of terrestrial plants: a meta-analysis. New Phytol. 2010;188:187-98. https://doi. org/10.1111/j.1469-8137.2010.03347.x.

7. Walther GR. Community and ecosystem responses to recent climate change. Philos T R Soc B. 2010;365:2019-24. https://doi.org/10.1098/rstb. 2010.0021.

8. Jin $B$, Li W, Jing $W$, Jiang $K Z$, Yang $W$, Jiang $X X, N i C Y$, Wang $Y L$, Teng $N$ J. The effect of experimental warming on leaf functional traits, leaf structure and leaf biochemistry in Arabidopsis thaliana. BMC Plant Biol. 2011;11:35. https://doi.org/10.1186/1471-2229-11-35.

9. Way DA, Yamori W. Thermal acclimation of photosynthesis: on the importance of adjusting our definitions and accounting for thermal 
acclimation of respiration. Photosynth Res. 2014;1 19:89-100. https://doi.org/ 10.1007/s11120-013-9873-7.

10. Glaubitz U, Li X, Schaedel S, Erban A, Sulpice R, Kopka J, Hincha DK, Zuther E. Integrated analysis of rice transcriptomic and metabolomic responses to elevated night temperatures identifies sensitivity- and tolerance-related profiles. Plant Cell Environ. 2017;40:121-37. https://doi. org/10.1111/pce.12850.

11. Pospišil P. Production of reactive oxygen species by photosystem II as a response to light and temperature stress. Front Plant Sci. 2016;7:1950. https://doi.org/10.3389/fpls.2016.01950.

12. Bita CE, Gerats T. Plant tolerance to high temperature in a changing environment: scientific fundamentals and production of heat stress-tolerant crops. Front Plant Sci. 2013;4:273. https://doi.org/10.3389/fpls.2013.00273.

13. Ohama N, Sato H, Shinozaki K, Yamaguchishinozaki K. Transcriptional regulatory network of plant heat stress response. Trends Plant Sci. 2017;22 53-65. https://doi.org/10.1016/j.tplants.2016.08.015

14. Niu Y, Xiang Y. An overview of biomembrane functions in plant responses to high-temperature stress. Front Plant Sci. 2018;9:915. https://doi.org/10. 3389/fpls.2018.00915.

15. Atkin OK, Loveys BR, Atkinson LJ, Pons TL. Phenotypic plasticity and growth temperature: understanding interspecific variability. J Exp Bot. 2006;57:26781. https://doi.org/10.1093/jxb/erj029.

16. Ashraf $\mathrm{M}$, Harris PJC. Photosynthesis under stressful environments: an overview. Photosynthetica. 2013;51:163-90. https://doi.org/10.1007/s11099013-0021-6.

17. Sakata S, Mizusawa N, Kubotakawai H, Sakurai I, Wada H. Psb28 is involved in recovery of photosystem II at high temperature in Synechocystis sp. PCC 6803. Biochim Biophys Acta. 2013;1827:50-9. https://doi.org/10.1016/j. bbabio.2012.10.004.

18. Allakhverdiev SI, Kreslavski VD, Klimov W, Los DA, Carpentier R, Mohanty P. Heat stress: an overview of molecular responses in photosynthesis. Photosynth Res. 2008;98:541-50. https://doi.org/10.1007/s11120-008-9331-0.

19. Patrick JW, Botha FC, Birch RG. Metabolic engineering of sugars and simple sugar derivatives in plants. Plant Biotechnol J. 2013;11:142-56. https://doi. org/10.1111/pbi.12002

20. Dumschott K, Richter A, Loescher W, Merchant A. Post photosynthetic carbon partitioning to sugar alcohols and consequences for plant growth. Phytochemistry. 2017;144:243-52. https://doi.org/10.1016/j.phytochem.2017. 09.019.

21. Lismont $C$, Nordgren M, Van Veldhoven PP, Fransen M. Redox interplay between mitochondria and peroxisomes. Front Cell Dev Biol. 2015;3:35. https://doi.org/10.3389/fcell.2015.00035.

22. Dietz KJ, Turkan I, Krieger-Liszkay A. Redox- and reactive oxygen speciesdependent signaling into and out of the photosynthesizing chloroplast. Plant Physiol. 2016;171:1541-50. https://doi.org/10.1104/pp.16.00375.

23. Sun $A Z$, Guo FQ. Chloroplast retrograde regulation of heat stress responses in plants. Front Plant Sci. 2016;7:398. https://doi.org/10.3389/fpls.2016.00398.

24. Grover A, Mittal D, Negi M, Lavania D. Generating high temperature tolerant transgenic plants: achievements and challenges. Plant Sci. 2013;205-206:3847. https://doi.org/10.1016/j.plantsci.2013.01.005.

25. Zhao J, He Q, Chen G, Wang L, Jin B. Regulation of non-coding RNAs in heat stress responses of plants. Front Plant Sci. 2016;7:1213. https://doi.org/ 10.3389/fpls.2016.01213.

26. Yoshida T, Ohama N, Nakajima J, Kidokoro S, Mizoi J, Nakashima K, et al. Arabidopsis HSFA1 transcription factors function as the main positive regulators in heat shock-responsive gene expression. Mol Gen Genomics. 2011;286:321-32. https://doi.org/10.1007/s00438-011-0647-7.

27. Srivastava R, Deng $Y$, Howell SH. Stress sensing in plants by an ER stress sensor/transducer, bZIP28. Front Plant Sci. 2014;5:59. https://doi.org/10.3389/ fpls.2014.00059.

28. Bäurle I. Plant heat adaptation: priming in response to heat stress. F1000research. 2016;5:694. https://doi.org/10.12688/f1000research.7526.1.

29. Lämke J, Brzezinka K, Altmann S, Bäurle I. A hit- and -run heat shock factor governs sustained histone methylation and transcriptional stress memory. EMBO J. 2016;35:162-75. https://doi.org/10.15252/embj.201592593.

30. Balasubramanian S, Sureshkumar S, Lempe J, Weigel D. Potent induction of Arabidopsis thaliana flowering by elevated growth temperature. PLoS Genet. 2006;2:e106. https://doi.org/10.1371/journal.pgen.0020106.

31. Flato G, Marotzke J, Abiodun B, Braconnot P, Chou SC, Collins WJ, et al. Evaluation of climate models. In: Stocker TF, Qin D, Plattner G-K, Tignor M, Allen SK, Boschung J, Nauels A, Xia Y, Bex V, Midgley PM, editors. Climate
Change. 2013: The physical science basis. contribution of working group I to the fifth assessment report of the intergovernmental panel on climate change, vol. 5. Cambridge: Cambridge University Press; 2013. p. 741-866.

32. Boyes DC, Zayed AM, Ascenzi R, Mccaskill AJ, Hoffman NE, Davis KR, Görlach J. Growth stage-based phenotypic analysis of Arabidopsis: a model for high throughput functional genomics in plants. Plant Cell. 2001;13:1499-510. https://doi.org/10.1105/tpc.13.7.1499.

33. Ren S, Ma K, Lu Z, Chen G, Cui J, Tong P, Wang L, Teng N, Jin B. Transcriptomic and metabolomic analysis of the heat-stress response of Populus tomentosa Carr. Forests. 2019;10:383. https://doi.org/10.3390/ f10050383.

34. Allen P, Bennett K, King J. PASW statistics by SPSS, a practical guide: version 18.0. Melbourne: Cengage Learning Press; 2010.

\section{Publisher's Note}

Springer Nature remains neutral with regard to jurisdictional claims in published maps and institutional affiliations.

Ready to submit your research? Choose BMC and benefit from:

- fast, convenient online submission

- thorough peer review by experienced researchers in your field

- rapid publication on acceptance

- support for research data, including large and complex data types

- gold Open Access which fosters wider collaboration and increased citations

- maximum visibility for your research: over $100 \mathrm{M}$ website views per year

At BMC, research is always in progress.

Learn more biomedcentral.com/submissions 